\title{
Vertical Handoff Decision Algorithm combined I mproved Entropy Weighting with GRA for Heterogeneous Wireless Networks
}

\author{
Shasha Zhao ${ }^{1,3, *}$, Fei Wang ${ }^{1,2}$, Yueqiang Ning ${ }^{1,2}$, Yi Xiao ${ }^{1,2}$, and Dengying Zhang ${ }^{1,3, *}$ \\ ${ }^{1}$ College of Internet of Things, Nanjing University of Posts and Telecommunications, \\ ${ }^{2}$ College of Telecommunications \& Information Engineering, \\ Nanjing University of Posts and Telecommunications, \\ ${ }^{3}$ Jiangsu Key Laboratory of Broadband Wireless Communication and Internet of Things, Nanjing University of \\ Posts and Telecommunications, \\ Nanjing, Jiangsu, 210003, China \\ [e-mail: zhaoss@njupt.edu.cn, zhangdy@njupt.edu.cn] \\ *Corresponding author: Shasha Zhao, Dengying Zhang
}

Received September 20, 2019; revised November 26, 2019; revised March 11, 2020; accepted March 20, 2020; published November 30, 2020

\begin{abstract}
Future network scenario will be a heterogeneous wireless network environment composed of multiple networks and multimode terminals (MMT). Seamless switching and optimal connectivity for MMT among different networks and different services become extremely important. Here, a vertical handoff algorithm combined an improved entropy weighting method based on grey relational analysis (GRA) is proposed. In which, the improved entropy weight method is used to obtain the objective weights of the network attributes, and GRA is done to rank the candidate networks in order to choose the best network. Through simulation and comparing the results with other vertical handoff decision algorithms, the number of handoffs and reversal phenomenon are reduced with the proposed algorithm, which shows a better performance.
\end{abstract}

Keywords: Heterogeneous wireless network, MMT, Vertical handoff, Improved entropy, GRA

This work is supported by Jiangsu province key research and development-industry foresight-competition project (BE2017111), the National Natural Science Foundation of China (61571241, 61872423), the Scientific Research Foundation of the Higher Education Institutions of Jiangsu Province, China (19KJB510050) and Postgraduate Educational and Teaching Reform Project of Jiangsu Province (JGLX19_045), Teaching Reform Research Project of Nanjing University of Posts and Telecommunications (JG01619JX29) 


\section{Introduction}

Rapid development of communication and information technology leads the network system and structure to be more and more complex. Flexible, quick, and accurate switching ability among different networks is required in the heterogeneous wireless networks, which is composed of multiple coexisting networks [1]. However, due to the differences in both network performance and terminal service, a single network can never meet all requirements of the users. Better network services to the multimode terminals (MMT) with various access networks coexisting and complementing each other becomes an inevitable development trend in the next-generation network. For the next generation wireless systems, it needs to embrace multiple wireless networks, including universal mobile telecommunications system (UMTS), 4G, wireless local area network (WLAN), etc, which is different in type, capacity, bandwidth, technology and so on. Meanwhile, for different service areas, the most important goal for MMT is to connect to the best network at all times [2]. It requires MMT to switch seamlessly among different networks. As a popular technology for heterogeneous network convergence, vertical handoff technology plays a decisive role in ensuring communication continuity and quality [3]. Therefore, a suitable vertical handoff algorithm will be greatly important for MMT, which is also a key issue in this work.

Vertical handoff technology, as an important factor for heterogeneous wireless network, ensures the MMT switch seamlessly among different underlying wireless networks. Over the last decade, heterogeneous networks have caused widespread concern and a large number of vertical handoff decision algorithms had been proposed [4-19]. To reduce the "ping-pong effect" and the switching delay, Received Signal Strength (RSS) method was proposed, while it results in the increase in packet loss rate [4-8]. Although the switching time can be determined with the comparison between the calculated dynamic RSS threshold and the RSS value of the existing network, reducing effectively the probability of handover failure, it also wastes network resources $[9,10]$. On the other hand, there is only one decision condition and a single problem of network attribute parameters considered in the network selection algorithm based on RSS.

In addition, according to the service type of the user and the state of the current network, the Markov decision model was established to perform handoff decision [11]. The overall performance of the heterogeneous network can be analyzed via a system simulation. The handoff decision had been made by using the transition probability of the Markov process to predict the state of the wireless network via constructing an expected total reward function during each connection, and maximizing the expected return in an iterative manner [12, 13]. Unfortunately, the Markov algorithm requires multiple iterations, and has relatively high complexity and large errors, due to its severe changes in the network parameters and instability. Furthermore, the algorithm is composed of fuzzy membership functions to quantify information without accurate description. As a result, complex and high switching 
delay still exist, although the fuzzy parameters can be established with multiple evaluation criteria [14].

However, considering the multiple attributes of the network, the heterogeneous wireless network vertical handoff algorithm needs a high accuracy of network selection. An improved multiplicative exponent weighting (MEW) algorithm is proposed for vertical handoff decision in heterogeneous wireless networks which meets the multi-attribute quality of service (QoS) requirement according to the traffic features [15]. While both the average user traffic cost and the number of vertical handoff are higher than others. Furthermore, for simple additive weighting (SAW), the total score of candidate network depends on the weighted sum of all attribute values [16]. It had been found that applying the analytic network process (ANP) and the enhanced Technique for Order Preference by Similarity to Ideal Solution (Topsis) to the vertical handoff algorithm is respectively helpful to obtain the standard weight and to rank the alternatives [17]. However, in the case of adding a network into, or deleting a network from the candidate network, it is easy to cause an abnormal ranking problem, affecting the switching performance. In order to improve ranking, a network selection algorithm combining Analytic Hierarchy Process (AHP) and GRA is proposed, where the AHP was used to calculate the weight of each network attribute, and GRA was applied to analyze and correlate the candidate networks [18, 19].

Overall, although some vertical handoff algorithms had been reported in the field of MMT, there are still two main weaknesses existed. One is that there is a large number of switching, and the other is the presence of reversal phenomenon. In this work, a vertical handoff decision algorithm based on the improved entropy method and the GRA algorithm is proposed. The improved entropy weight method is applied to obtain the weight of each attribute. The GRA is used to rank the available networks. Simulation results show that both the number of handoff and the reversal phenomenon were significantly reduced with this vertical handoff decision algorithm.

The structure of this paper is organized as follows. The methodology including the system model and the vertical handoff decision algorithm is respectively shown in Section 2 and Section 3. The simulation results and discussions are shown in Section 4, and the Section 5 is composed of the conclusions.

\section{System Model}

A scenario of the heterogeneous wireless network is firstly assumed, where the MMT is located in an area with UMTS1, UMTS2, 4G, and WLAN networks as shown in Fig. 1. Among different service areas, the MMT can access to different types of candidate networks. 


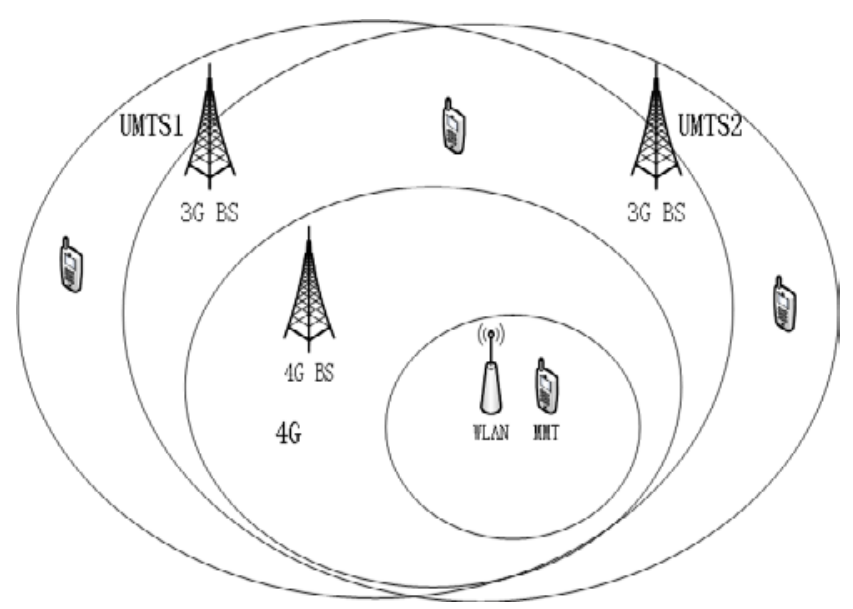

Fig. 1. The scenario of the heterogeneous wireless network used in this work.

For the scenario of heterogeneous wireless networks, it is composed of $m$ networks that defined as $a=[1,2, \ldots, m]$. Meanwhile, there exist $n$ attributes that defined as $b=[1,2, \ldots, n]$, including cost per byte (CB), security (S), available bandwidth (AB), packet delay (D), packet jitter (J), and packet loss (L). The improved entropy weight method is applied to assign weight to each attribute. In order to make each attribute parameter to be more reasonable, the result analysis was done with considering the subjective and objective weight. The combination weight is defined as $w_{b}$. The degree of association $r_{a}$ between the evaluation network and the ideal network is deduced via the GRA method with the function expressed as

$$
r_{a}=\frac{1}{n} \sum_{b=1}^{n} w_{b} \zeta_{a b} \quad a=1,2, . ., m
$$

where $\zeta_{a b}$ denotes the correlation coefficient. The value of $r_{a}$ is bigger, the performance of the candidate network is better. Finally, through ranking the candidate networks by $r_{a}$, and the best candidate network that with the biggest value of $r_{a}$ was chosen.

\section{Vertical Handoff Decision Algorithm}

In this section, the data preprocessing is described at first, and then the improved Entropy Weighting Method is done to obtain the objective weight of each attribute. Next, the composite weight is determined by combining the objective weight with subjective weight. Finally, the GRA is used to derive the degree of association between the evaluation object and the ideal object. The best candidate network will be chosen according to the degree value.

\subsection{Data Preprocessing}

It is assumed that there are $m$ candidate networks, and each candidate network contains $n$ evaluation attributes. Thus, the decision matrix $X$ is established as 


$$
X=\left[x_{a b}\right]_{m \times n}=\left[\begin{array}{ccccc}
x_{11} & x_{12} & \cdots & \cdots & x_{1 n} \\
x_{21} & x_{22} & \cdots & \cdots & x_{2 n} \\
\vdots & \vdots & \ddots & & \vdots \\
\vdots & \vdots & & \ddots & \vdots \\
x_{m 1} & x_{m 2} & \cdots & \cdots & x_{m n}
\end{array}\right] \quad a=1,2 \ldots, m, \quad b=1,2, \ldots, n
$$

where $x_{a b}$ denotes the $b_{t h}$ attribute of the $a_{t h}$ network.

Standardize the data $x_{a b}$. For bigger and better parameters, the attribute parameter standardized value $y_{a b}$ can be determined with the standardization formula

$$
y_{a b}=\frac{x_{a b}-\min _{a}\left(x_{a b}\right)}{\max _{a}\left(x_{a b}\right)-\min _{a}\left(x_{a b}\right)} a=1,2, \ldots, m, b=1,2, \ldots, n
$$

However, for smaller and better parameters, the standardization formula will be deduced as

$$
y_{a b}=\frac{\max _{a}\left(x_{a b}\right)-x_{a b}}{\max _{a}\left(x_{a b}\right)-\min _{a}\left(x_{a b}\right)} \quad a=1,2, \ldots, m, b=1,2, \ldots, n
$$

where $\max _{a}\left(x_{a b}\right)$ denotes the maximum value of the $b_{t h}$ attribute and $\min _{a}\left(x_{a b}\right)$ denotes the minimum value of the $b_{t h}$ attribute. Then the standard matrix $Y$ can be expressed as

$$
Y=\left[\begin{array}{ccccc}
y_{11} & y_{12} & \cdots & \cdots & y_{1 n} \\
y_{21} & y_{22} & \cdots & \cdots & y_{2 n} \\
\vdots & \vdots & \ddots & & \vdots \\
\vdots & \vdots & & \ddots & \vdots \\
y_{m 1} & y_{m 2} & \cdots & \cdots & y_{m n}
\end{array}\right]
$$

\subsection{Entropy Weighting Method}

As an objective weighting method, entropy weight is widely used in multi-objective selection and various evaluations to determine the weight of attributes.

1) As for the traditional entropy method, the entropy $P_{b}$ of the $b_{t h}$ attribute is defined as $P_{b}=-t \sum_{b=1}^{n} r_{a b} \ln r_{a b}$, where $t=1 / \ln n, r_{a b}$ denotes the proportion of the $b_{t h}$ attribute for the $a_{t h}$ network which described as $r_{a b}=y_{a b} / \sum_{n=1}^{b} y_{a b}$. Nevertheless, it is unreasonable, when $r_{a b}=0$. Therefore, the $r_{a b}$ is changed here as

$$
r_{a b}=\frac{y_{a b}+0.1}{\sum_{b=1}^{n} y_{a b}+0.1}
$$


2) As a consequence, the entropy of the $b_{t h}$ attribute can be described as

$$
P_{b}=-t \sum_{b=1}^{n} r_{a b} \ln r_{a b}, \quad r_{a b}=\frac{y_{a b}+0.1}{\sum_{b=1}^{n} y_{a b}+0.1}
$$

3) In general, the smaller information entropy of an attribute is, the more information is provided and the greater value the weight has. Therefore, the variation coefficient $e_{b}$ is described as $e_{b}=1-P_{b}$. Then the greater value $e_{b}$ has, the higher the importance of the attribute will be.

4) By normalizing $e_{b}$, the objective weight for each attribute can be deduced as

$$
w_{b}^{\prime}=\frac{e_{b}}{\sum_{b=1}^{n} e_{b}}, b=1,2, \ldots, n
$$

5) Determine the composite weight. The reasonable combination of the subjective and objective weight of attributes is a key factor for making multi-attribute decision. The subjective weight of the attribute can be described as $w^{\prime \prime}=\left(w_{1}{ }^{\prime \prime}, w_{2}, \ldots, w_{n}{ }^{\prime \prime}\right)^{T}$, where $0 \leq w_{b}^{\prime \prime} \leq 1, b=1,2, \ldots n, \sum_{b=1}^{n} w_{b}{ }^{\prime \prime}=1$ [17]. The objective weight that defined as $w^{\prime}=\left(w_{1}^{\prime}, w_{2}^{\prime}, \ldots, w_{n}^{\prime}\right)$ can be obtained via the improved Entropy weight method, where $0 \leq w_{b}{ }^{\prime} \leq 1, b=1,2, \ldots n, \sum_{b=1}^{n} w_{b}{ }^{\prime}=1$. The composite weight will be given as

$$
w_{b}=\frac{w_{b} w_{b}^{\prime \prime}}{\sum_{b=1}^{n} w_{b} w_{b}^{\prime \prime}}, \quad b=1,2, \ldots, n
$$

\subsection{Grey Relational Analysis Method}

The GRA method can provide a quantitative character of the development and change situation of a system, which is suitable for dynamic process analysis. The similarity or dissimilarity of the development trends among different attributes is used to decide the degree of association between the evaluation and the ideal object. The candidate network mentioned here is defined as evaluation object. The best network will be chosen by comparing the degree of association between the candidate and the ideal network. The larger the degree of association has, the better the candidate network will be. Furthermore, the specific progress is shown as below.

1) The reference sequence is an ideal comparison criterion, which is formed from the 
optimal value of each index and expressed as

$$
y_{b}^{*}=\left\lfloor y_{1}^{*}, y_{2}^{*}, \cdots, \cdots, y_{n}^{*}\right\rfloor \quad b=1,2, \ldots, n
$$

2) Here, the physical meaning of each attribute in the system is different, and the dimension of the sequence is not necessarily the same. With the gray correlation analysis, the dimensionless sequence processing will be generally performed and defined as

$$
y_{a b}^{\prime}=\frac{y_{a b}}{\frac{1}{n} \sum_{b=1}^{n} y_{a b}}
$$

3) The correlation coefficient will be deduced as

$$
\begin{aligned}
& \zeta_{a b}=\frac{\min _{a} \min _{b}\left|y_{b}^{*}-y_{a b}^{\prime}\right|+\rho \max _{a} \max _{b}\left|y_{b}^{*}-y_{a b}^{\prime}\right|}{\left|y_{b}^{*}-y_{a b}^{\prime}\right|+\rho \max _{a} \max _{b}\left|y_{b}^{*}-y_{a b}^{\prime}\right|}, \\
& a=1,2, \ldots, m, b=1,2, . ., n
\end{aligned}
$$

where $\left|y_{b}^{*}-y_{a b}^{\prime}\right|$ is the absolute value of $y_{b}^{*}$ and $y_{a b}^{\prime}$, and $\rho$ denotes the resolution coefficient, satisfying $0<\rho<1$. The smaller the $\rho$ is, the greater the difference between the correlation coefficients will be. However, $\rho$ is usually taken as 0.5 .

4) As a result, the gray weighted association can be expressed as

$$
r_{a}=\frac{1}{n} \sum_{b=1}^{n} w_{b} \zeta_{a b}
$$

where $r_{a}$ denotes the degree of relevance between the evaluation object and the ideal object, and $w_{b}$ can be obtained from equation (9). Thus, based on the value of $r_{a}$, the candidate networks will be ranked, and the best access network with the biggest one will be chosen.

\subsection{Select the Best Network}

Based on the vertical handoff algorithm formulation above, the optimal network selection algorithm is described as following process:

\section{1: Data preprocessing:}

2: Set $y_{a b}=\frac{x_{a b}-\min _{a}\left(x_{a b}\right)}{\max _{a}\left(x_{a b}\right)-\min _{a}\left(x_{a b}\right)}$ or $y_{a b}=\frac{\max _{a}\left(x_{a b}\right)-x_{a b}}{\max _{a}\left(x_{a b}\right)-\min _{a}\left(x_{a b}\right)}$.

3: loop for each decision slot:

4: $\quad$ determine the composite weight and the correlation coefficient: $w_{a}, \zeta_{a b}$

5: compute the gray weighted association: $r_{a}$

6: $\quad$ compare $r_{a}$ of four networks

7: $\quad$ if the $a_{t h}$ network owns the biggest $r_{a}$

8: $\quad$ select it as the best access network 
9: $\quad$ end if

10: end loop

\section{Simulation Results and Analysis}

\subsection{Simulations Condition}

To demonstrate and verify the advantage of our algorithm on network handoffs, as a comparison, other algorithms including Markov [13], MEW [15], SAW [16], AHP and GRA [18], and Topsis [17], were chosen to do simulation at the same time. The attribute parameters that considered in the simulation are CB, S, AB, D, J, and L. Four traffic classes are considered which includes background, conversational, interactive and streaming. Besides the values concerning each attribute for each network are shown in Table 1, the subject weights for traffic classes are given respectively by Ref. [17].

Table 1. Values of attributes for each candidate network [17]

\begin{tabular}{|c|c|c|c|c|c|c|}
\hline $\begin{array}{c}\text { attribute } \\
\text { network }\end{array}$ & $\mathbf{C B}(\mathbf{\% )})$ & $\mathbf{S ( \% )}$ & $\mathbf{A B}(\mathbf{m b p s})$ & $\mathbf{D}(\mathbf{m s})$ & $\mathbf{J}(\mathbf{m s )}$ & $\mathbf{L}(\mathbf{p e r 1 0})$ \\
\hline UMTS1 & 60 & 50 & $0.1-2$ & $20-50$ & $5-15$ & $20-80$ \\
\hline UMTS2 & 80 & 70 & $0.1-2$ & $30-60$ & $10-20$ & $25-90$ \\
\hline WLAN & 15 & 60 & $1-10$ & $100-140$ & $10-20$ & $20-80$ \\
\hline 4G & 50 & 60 & $1-60$ & $60-140$ & $3-10$ & $20-80$ \\
\hline
\end{tabular}

\subsection{Results and Analysis}

With the simulation done by the MATLAB simulator, the number of handoffs is derived. With regard to the number of handoffs, the whole algorithms were run in 50, 100 and 150 vertical handoff decision points, respectively. To ensure the reliability of the results for reversal phenomenon, the whole algorithms were run in 200 vertical handoff decision points.

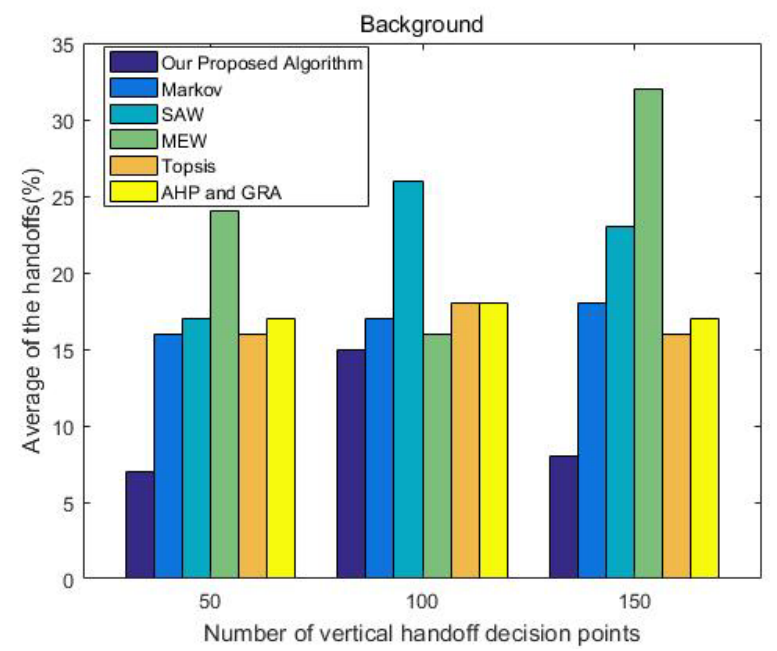

Fig. 2. Average of the handoffs for the traffic class of Background. 


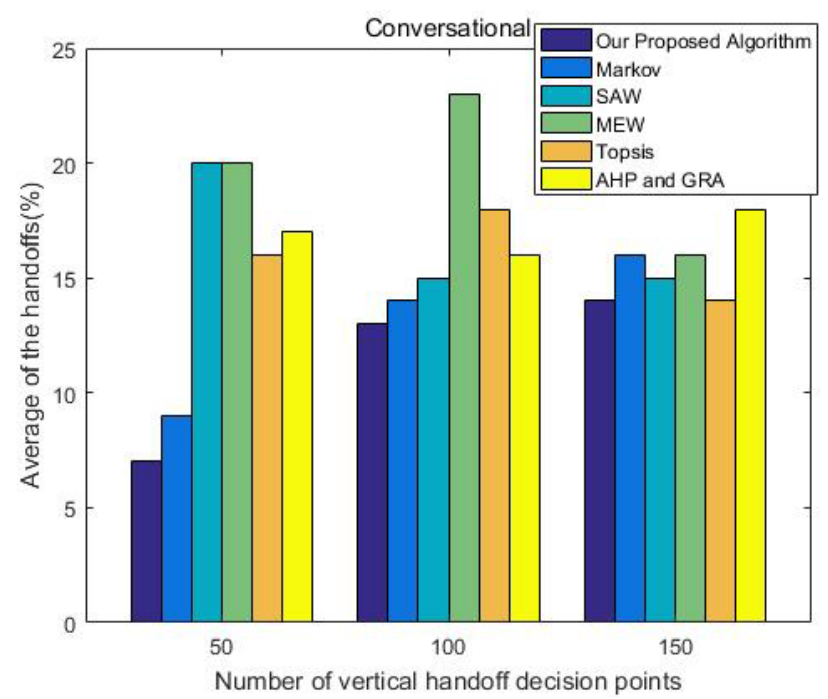

Fig. 3. Average of the handoffs for the traffic class of Conversational.

The average values rates of handoffs for our and other five decision algorithms according to the four traffic classes are respectively displayed in Figs. 2-5. Obviously, the average switching rates of our proposed algorithm are not only lower than $20 \%$, but also less than any one of the rest algorithms. In fact, due to the higher number of handoffs, SAW and MEW are poorly for network selection. As shown in Fig. 2, the average switching rate of SAW and MEW is respectively $23 \%$ and $32 \%$, which performs over $8 \%$ greater than that of our proposed algorithm under 150 decision points. Although Topsis performs better than SAW and MEW for the lower number of handoffs, as displayed in Fig. 3, it also performs worse than our proposed algorithm. As shown in Fig. 4, our algorithm and Topsis both present a close average switching rate of $15 \%$ for the 50 decision points. Nevertheless, with our algorithm, a lower value of $13 \%$ compared with that of $18 \%$ resulted from the Topsis with 100 decision points is obvious. Besides, the algorithm of AHP and GRA performs a little worse than our proposed algorithm for its higher value of average of the handoffs because it just considers the subjective aspect without the objective aspect which is not comprehensive. Although the algorithm of Markov presents lower average of handoffs than our algorithm under 50 decision points, it performs higher average of handoffs under 100 and 150 decision points. Therefore, our algorithm can reduce the number of vertical handoffs more effectively, regardless of the number of vertical handoff decision points used.

Fig. 5 shows the average of the handoffs for the traffic class of interactive. In detail, the number of average switching rates for the interactive class is relatively larger comparing with other three traffic classes. On the contrary, the conversational class performs the best with a lower average switching rate among the four traffic classes as shown in Fig. 3, which may be attributed to the high weight of delay of the interactive class, further reducing the impact of other attributes. In the future, the improvement of the vertical handoffs algorithms 
could be done by adding more effective decision attributes reasonably.

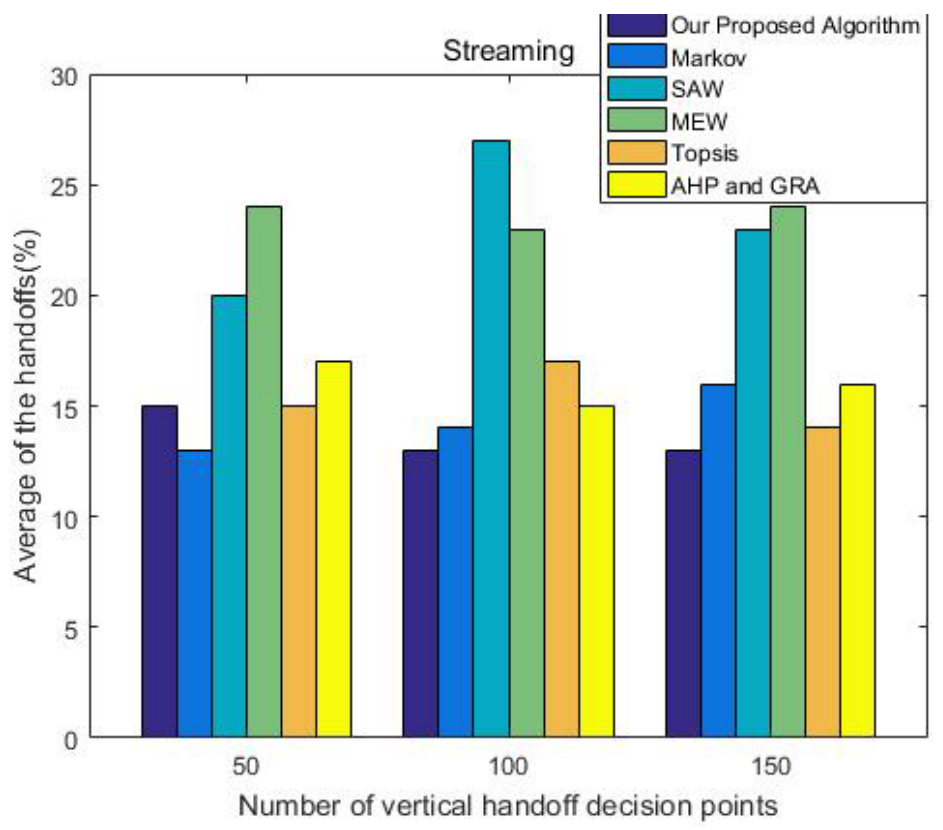

Fig. 4. Average of the handoffs for the traffic class of streaming.

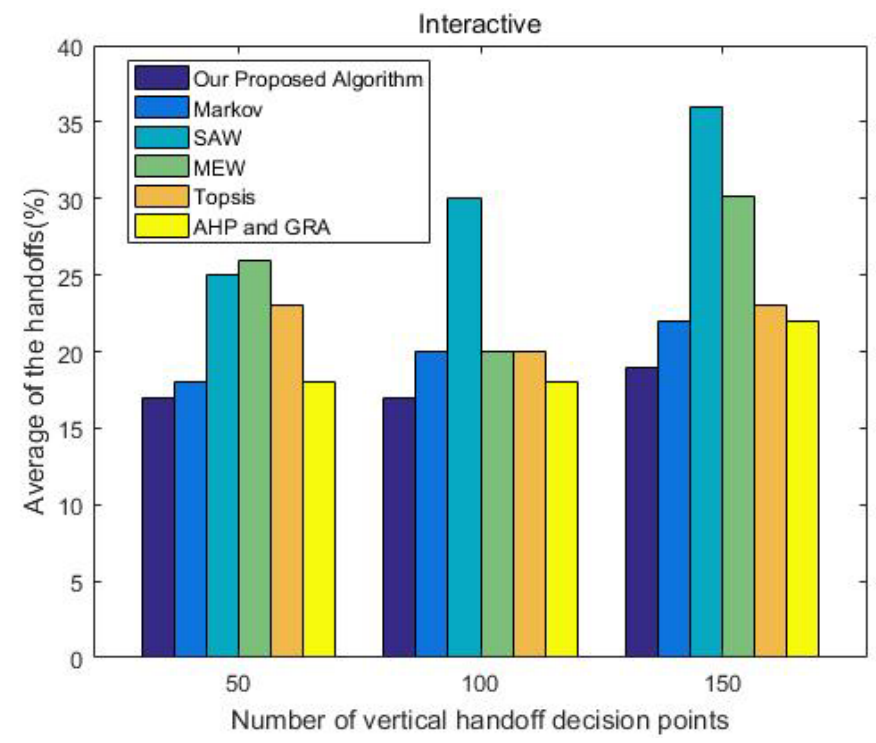

Fig. 5. Average of the handoffs for the traffic class of interactive.

Fig. 6 implies the average of reversal phenomenon for the four traffic classes. Notably, our proposed algorithm presents the lowest average of reversal phenomenon with a value of $10 \%, 12 \%, 16 \%$ and $13 \%$, while the MEW presents the highest one of $25 \%, 25 \%, 24 \%$ and $31 \%$ for background, conversational, interactive and streaming, respectively. This suggests that MEW is terrible for solving the problem of vertical handoff. Actually, the whole values 
provided by our proposed algorithm are lower than those of derived from the algorithms of Markov, SAW, MEW, AHP and GRA, and Topsis, indicating an outstanding of our proposed algorithm in reduction of the reversal phenomenon.

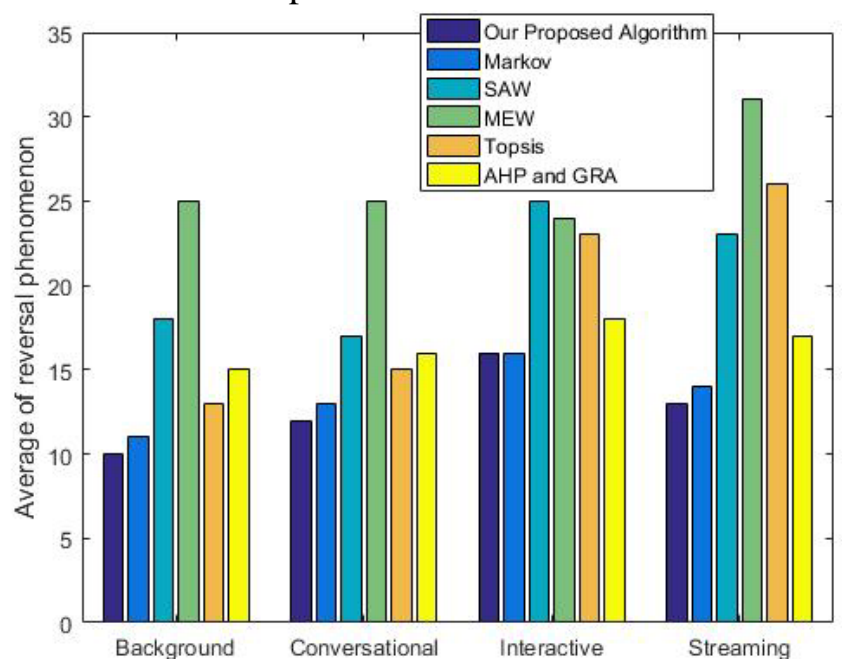

Fig. 6. The average of reversal phenomenon for all traffic classes

\subsection{Analysis of Computational Complexity}

Here, the computational complexity is calculated based on the size of the input of the algorithm (i.e. the number of data) [20]. Both the algorithmic complexity functions and the corresponding valuations used here are listed in Table 2, and the derived results are listed in Table 3. As shown in Table 3, on the one hand, the algorithm of Markov presents the highest value of computational complexity because it requires multiple iterations.On the other hand, the computational complexity of our proposed algorithm is the same as that of MEW, AHP and GRA, and Topsis, while it is larger than that of SAW. The higher complexity may be attributed to the addition of data preprocessing used here.

Table 2. Algorithmic complexity functions and the corresponding valuation [20].

\begin{tabular}{|c|c|c|}
\hline Notation & Name & Numerical value \\
\hline $\mathrm{O}(1)$ & Constant order & 10 \\
\hline $\mathrm{O}(\log \mathrm{n})$ & Logarithmic order & 15 \\
\hline $\mathrm{O}(\mathrm{n})$ & Linear order & 20 \\
\hline $\mathrm{O}(\mathrm{n} 2)$ & Quadratic & 25 \\
\hline $\mathrm{O}(\mathrm{n} 3)$ & Cubic order & 30 \\
\hline
\end{tabular}

Table 3. Computational complexity of all algorithms.

\begin{tabular}{|c|c|c|c|c|c|c|}
\hline Algorithm & $\begin{array}{c}\text { This } \\
\text { work }\end{array}$ & Markov & SAW & MEW & Topsis & $\begin{array}{c}\text { AHP and } \\
\text { GRA }\end{array}$ \\
\hline $\begin{array}{c}\text { Computational } \\
\text { complexity }\end{array}$ & 25 & 30 & 20 & 25 & 25 & 25 \\
\hline
\end{tabular}


Table 4. The correspondence between the abbreviation and the full name

\begin{tabular}{|c|c|}
\hline Abbreviations & Full Name \\
\hline MMT & multimode terminals \\
\hline GRA & grey relational analysis \\
\hline UMTS & universal mobile telecommunications system \\
\hline WLAN & Received Signal Strength \\
\hline RSS & quality of service \\
\hline QoS & simple additive weighting \\
\hline SAW & analytic network process \\
\hline ANP & Analytic Hierarchy Process \\
\hline Topsis & Technique for Order Preference by Similarity to Ideal Solution \\
\hline AHP & cost per byte \\
\hline CB & security \\
\hline S & available bandwidth \\
\hline AB & packet delay \\
\hline D & packet jitter \\
\hline J & packet loss \\
\hline L &
\end{tabular}

\section{Conclusion}

In this paper, a vertical handoff algorithm based on improved entropy weighting combined with GRA was proposed for heterogeneous wireless networks. Specifically, the improved entropy weight method is used to get the objective weights of network attributes. The GRA method is applied to rank the candidate networks for selecting the best candidate network. The numerical simulation results show that our algorithm can reduce the number of handoffs and the reversal phenomenon effectively, and obtain better performance compared with other algorithms including Markov, SAW, MEW, AHP and GRA and Topsis. Although our proposed algorithm demonstrates a bigger computational complexity than that of SAW, a same value is also observed for the rest algorithm, which may be caused by the addition of data preprocessing used here. Also, in future, we can research the vertical handoff algorithms for different traffic classes by adding more effective decision attributes such as network cost and speed, etc. It may provide a more effective vertical handoff among different networks for MMT. 


\section{References}

[1] Udhayakumar S, Raja Kumar R, Indumathi P, "Network selection in wireless heterogeneous network," in Proc. of International Conference on Recent Trends in Electrical, Control and Communication (RTECC), pp.57-60, 2018. Article (CrossRef Link)

[2] Gustafsson E, Jonsson A, “Always best connected,” IEEE Wireless communications, vol. 49, no. 1, pp.49-55, 2003. Article (CrossRef Link)

[3] Zekri M, Jouaber B, Zeghlache D, “A review on mobility management and vertical handover solutions over heterogeneous wireless networks,” Computer Communications, vol. 35, no. 17, pp.2055-2068, 2012. Article (CrossRef Link)

[4] Madaan J, Kashyap I, "Vertical handoff with predictive received signal strength in next generation wireless network,” Computer Network and Information Security, vol. 8, no. 8, pp. 27-38, August 2016. Article (CrossRef Link)

[5] Panda A, Patra S K, Acharya D P, "Received Signal Strength based vertical hand off scheme for K-Tier heterogeneous networks" in Proc. of International Conference on Communication Systems and Network Technologies. IEEE, pp.327-331, 2013. Article (CrossRef Link)

[6] Ali M, Saquib M, “Analytical framework for WLAN-cellular voice handover evaluation,” IEEE Trans. Mobile Comput., vol. 12, no. 3, pp. 447-460, Mar. 2013. Article (CrossRef Link)

[7] Ylianttila M, Makela J, Pahlavan K, "Analysis of handoff in allocation-aware vertical multi-access network,” Computer Networks, vol. 47, no. 2, pp.185-201,2005. Article (CrossRef Link)

[8] Pahlavan K, Krishnamurthy P, Hatami A, Ylianttila M, Makela J, Pichna R, Vallstron J, "Handoff in hybrid mobile data networks,” IEEE Personal Communication, vol. 7, no.2, pp.34-47, 2000. Article (CrossRef Link)

[9] Li D, Li E V, Van de Capelle A, "Improving slow-start based probing mechanisms for flow adaptation after handovers,” Computer Networks, vol. 56, no. 1, pp.329-344, 2000.

[10] Mohanty S, Akyildiz I, "A cross-layer (layer 2+3) handoff management protocol for next-generation wireless systems,” IEEE Transaction on Mobile Computing, vol. 5, no. 10, pp. 1347-1360, 2006. Article (CrossRef Link)

[11] Chen L, Li H, “An MDP-based vertical handoff decision algorithm for heterogeneous wireless networks," in Proc. of Wireless Communications and Networking Conference (WCNC), 2016 IEEE, pp.1-6, 2016.

[12] Ma B, Deng H, Xie X Z, Liao X F, “An optimized vertical handoff algorithm based on Markov Process in vehicle heterogeneous network,” China Communications, vol.12, no.4, pp.106-116, 2015.Article (CrossRef Link)

[13] Ning Z L, Song Q Y, Liu Y J, "Markov-Based vertical handoff decision algorithms in heterogeneous wireless networks,” Computers \& Electrical Engineering, vol. 40, no. 2, pp.456-472, 2013. Article (CrossRef Link)

[14] Tansu F, Salamah M, "On the vertical handoff decision for wireless overlay networks. computer networks, International Symposium,” in Proc. of 2006 International Symposium on Computer Networks, pp.111-115, 2006. Article (CrossRef Link)

[15] Liu S M, Pan S, Mi Z K, Meng Q Mn, Xu M H, “An improved multiplicative exponent weighting vertical handoff algorithm for WLAN/WCDMA heterogeneous wireless networks," Engineering Sciences, vol. 10, no. 1, pp.86-90, 2012. Article (CrossRef Link)

[16] Savitha K, Chandrasekar C, "Vertical Handover decision schemes using SAW and WPM for Network selection in Heterogeneous Wireless Network,” Global Journal of Computer Science and Technology, vol. 11, no. 9, pp. 1-7, May 2011. Article (CrossRef Link)

[17] Mohamed L, Leghris C, Abdellah A, “An Enhanced-Topsis based network selection technique for next generation wireless networks," in Proc. of the 20th International Conference on Telecommunications, pp. 1-5, 2013. Article (CrossRef Link)

[18] Verma R, Singh N P, “GRA based network selection in heterogeneous wireless networks," Wireless personal communications, vol. 72, no. 2, pp.1437-1452, 2013. Article (CrossRef Link) 
[19] Sahana B, Rohin D, "Multi-criteria vertical handoff decision algorithm using hierarchy modeling and additive weighting in an Integrated WiFi/WiMAX/UMTS environment," KSII Transactions on Internet and Information Systems, vol. 8, no.1, pp. 35-57, January 29, 2014.

Article (CrossRef Link)

[20] T. Cormen, C. Leiserson, R. Rivest, and C. Stein, Introduction to algorithms, 2nd edition, The MIT Press, September 2001.

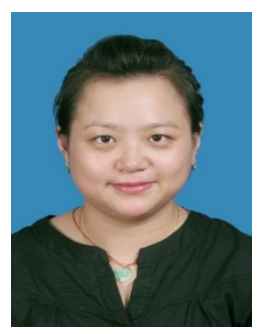

Shasha Zhao received the B.E. degree in electrical engineering from Anhui Normal University, Wuhu, in 2004, and received the Ph.D. degrees from Nanjing University of Posts and Telecommunication, Nanjing, China, in 2013. She is currently a lecturer of the School of Internet of Things, Nanjing University of Posts and Telecommunication, Nanjing, China. Her research interests mainly focus on cognitive wireless network, wireless network virtualization and Internet of Things technology.

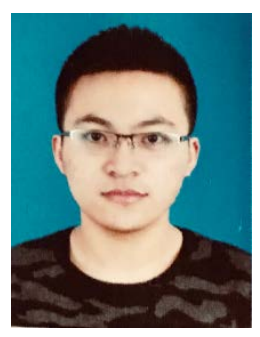

Fei Wang received the B.E. degree in electrical engineering from South East University Chengxian College, Nanjing, in 2004. He is currently working towards his M.S. degree in the College of Telecommunications \& Information Engineering, Nanjing University of Posts and Telecommunications, Nanjing. His research interests mainly focus on the area of wireless communications and networking, and vertical handoff in heterogeneous wireless network.

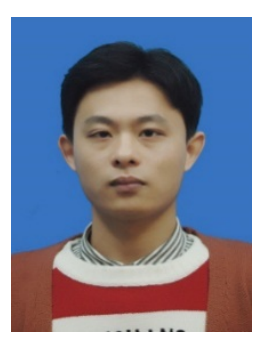

Yueqiang Ning received the B.E. degree in communication engineering from West Anhui University, AnHui, in 2017. He is currently working towards his M.S. degree in the College of Telecommunications \& Information Engineering, Nanjing University of Posts and Telecommunications, Nanjing. His research interests mainly focus on the area of resource allocation in network function virtualization.

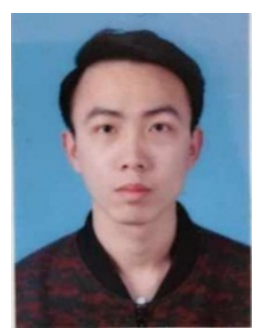

Xiao Yi received the B.E degree in communication engineering from Nanyang Institute of Technology, Nanyang, in 2017. He is currently working towards his M.S. degree in the College of Communications \& Information Engineering, Nanjing University of Posts and Telecommunications, Nanjing. His research interests are focused on the area of machine learning and computer network.

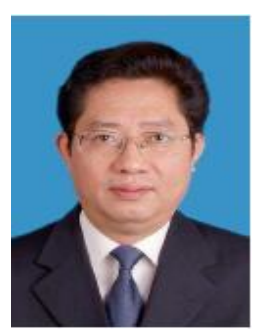

Dengying Zhang (M'17) received the B.S., M.S., and Ph.D. degrees from Nanjing University of Posts and Telecommunication, Nanjing, China, in 1986, 1989 and 2004, respectively. He is currently a Professor of the School of Internet of Things, Nanjing University of Posts and Telecommunication, Nanjing, China. He was a visiting scholar in Digital Media Lab, Umea University, Sweden, from 2007 to 2008. His research interests include signal and information processing, networking technique, and information security. 